\title{
Transpapillary gallbladder drainage: when and how?
}

\section{다)(우우}

\author{
Authors \\ Atsushi Sofuni, Takao Itoi \\ Institution \\ Department of Gastroenterology and Hepatology, Tokyo \\ Medical University, Tokyo, Japan \\ Bibliography \\ DOI https://doi.org/10.1055/a-0838-5456 | \\ Endoscopy International Open 2019; 07: E1115-E1116 \\ (c) Georg Thieme Verlag KG Stuttgart · New York \\ elSSN 2196-9736
}

\author{
Corresponding author \\ Takao Itoi, MD PhD, Department of Gastroenterology and \\ Hepatology, Tokyo Medical University, 6-7-1 Nishishinjuku, \\ Shinjuku-ku, Tokyo 160-0023, Japan \\ Fax: +81-3-53816654 \\ itoi@tokyo-med.ac.jp
}

According to Tokyo Guidelines 2018, the basic treatment for acute cholecystitis (AC) is cholecystectomy [1-4]. However, there are several risks associated with emergency surgery, and depending on the capabilities of surgeons and anesthesiologists in some hospitals, it is not always possible to perform an emergency or early operation. In such a case, gallbladder drainage is selected as a palliative treatment. Cholecystectomy is finally recommended for cases of cholecystectomy drainage or conservative treatment.

For general initial treatment of AC, fasting, infusion, and antibiotics are administered, and analgesics are administered if the pain is strong. Tokyo Guidelines 2018 (TG18) gives a severity treatment strategy for AC [1,2]. In mild (Grade I) AC, TG18 recommends early laparoscopic cholecystectomy (Lap-C) if the patients meet the criteria of Charlson comorbidity index $(\mathrm{CCl})$ $\leq 5$ and the American Society of Anesthesiologists physical status classification (ASA-PS) $\leq 2$. Grade II (moderate) AC is often accompanied by severe local inflammation. Therefore, surgeons should take the difficulty of cholecystectomy into consideration in selecting a treatment method. If patients meet the criteria of $\mathrm{CCl} \leq 5$ and ASA-PS $\leq 2$, TG18 recommends early Lap-C performed by experienced surgeons; and if not, after medical treatment and/or gallbladder drainage, Lap-C would be indicated. In severe (Grade III) AC, systemic management for accompanying organ failure is needed. These are that the patients have favorable organ system failure (FOSF) and negative predictive factors, who meet the criteria of $\mathrm{CCl} \leq 3$ and ASA-PS $\leq 2$, and who are being treated at an advanced center (where experienced surgeons practice). If the patient is not considered suitable for early surgery, TG18 recommends early/ urgent biliary drainage followed by delayed Lap-C once the patient's overall condition has improved.

The indication for gallbladder drainage, as mentioned above, follows the strict criteria of TG18; briefly, when the patient's general condition is poor and unable to bear anesthesia, when the condition does not respond to mild and conservative treatment, and when an emergency operation cannot be performed due to the problem of the capability of the surgeon/anesthesiologist, then gallbladder drainage is selected. Gallbladder drainage improves pain and promptly improves inflammation. Methods of gallbladder drainage include percutaneous drainage and endoscopic drainage. Percutaneous drainage includes percutaneous transhepatic gallbladder aspiration (PTGBA) and percutaneous transhepatic drainage (PTGBD). Endoscopic drainage includes endoscopic transpapillary gallbladder drainage and EUS-guided gallbladder drainage (EUS-GBD). TG18 recommends PTGBD as a standard drainage method for surgically high risk patients with $\mathrm{AC}$ [3]. As a result of an international multicenter comparative study on the treatment of AC, Itoi et al. [5] reported that PTGBD showed similar clinical efficacy compared with endoscopic gallbladder drainage including EUS-GBD and endoscopic transpapillary gallbladder drainage without a significant increase in complication rate.

Endoscopic transpapillary gallbladder drainage can be divided into two different methods: endoscopic naso-gallbladder drainage (ENGBD) and endoscopic gallbladder stenting (EGBS). These transpapillary procedures are used to place a drainage tube in the gallbladder via the cystic duct in the same manner as with endoscopic retrograde cholangiopancreatography (ERCP) [6]. Before this, endoscopic transpapillary gallbladder drainage was used for gallbladder cytology, etc. Recently, it has become a procedure that is being used as one of the treatment options for AC. The difficulty is that the success rate is not necessarily high as the approach requires specialist knowledge and experience. However, it is an effective procedure in patients with ascites, severe coagulopathy, thrombocytopenia, or an anatomically inaccessible location [7]. In addition to the procedural difficulty, another problem with this approach is 
the risk of cystic duct perforation. The cystic duct may also cause edematous changes in situations where inflammation of the gallbladder is spreading to the cystic duct. In such a situation, the cystic duct wall is very weak and there is a tendency for perforation by the guidewire to occur easily. According to TG18, endoscopic transpapillary gallbladder drainage is supposed to be performed at facilities with skilled endoscopists in high volume centers [3]. Considering situations where preventive anticoagulants and antiplatelet agents are administered, endoscopic transpapillary gallbladder drainage occupies an important position when gallbladder drainage is required during AC. Moreover, it is expected that its success rate will increase as a result of improvements in endoscopic treatment devices.

Redesigned plastic stents are expected to be developed for AC. The novel stent in the paper by Nakahara et al. [8] has a unique structure and shape. The tip of the stent has a three-dimensional spiral-shaped structure, and there are sideholes inside the spiral, compared to conventional double-pigtail type stents. This is expected to prevent migration of the stent and, even if the stent adheres to the constricted gallbladder wall, drainage will be maintained without the sideholes being blocked. The shaft of the stent is semicircular and fits the anatomical shape of the cystic duct, and the sidehole of the shaft is designed to drain the bile from the common bile duct. The distal side of the stent is straight, with a flap to prevent proximal migration. The novel stent is reported to have significantly lower rates of late adverse events and stent migration compared to earlier stents. Although this paper was a retrospective study, this novel stent may be effective for $A C$ and a randomized controlled trial is expected shortly.

Regarding the choice of ENGBD or EGBS, according to a randomized controlled trial by Itoi et al. with ENGBD and EGBS groups [9], the procedure success rate was $92 \%$ in the ENGBD group and $86 \%$ in the EGBS group, and the clinical response rates were $87 \%$ and $78 \%$, respectively with no significant differences between the two groups. There was also no significant difference between the two groups for procedure time or adverse events. Therefore, ENGBD and EGBS are useful and confirmed to be safe, and either ENGBD or EGBS may be considered for gallbladder drainage based on the patient's background and the endoscopist's decision [3].

The procedure for endoscopic gallbladder drainage [6] is as follows: 1. Deep bile duct cannulation from the papilla under ERCP. 2. Visualize the cystic duct by cholangiography. There are possible variations due to the combination of the diameter of the common bile duct, the number and size of gallstones compressing the cystic duct, the orientation of the cystic duct, etc. Considering these variations, several tries may be necessary to find the cystic duct with a guidewire. 3 . Find a helical cystic duct with a hydrophilic guidewire and carefully advance the guidewire. 4. Advance the guidewire to the deep portion of the gallbladder. 5. Allow the catheter to follow the gallbladder along the guidewire. 6 . Replace the hydrophilic guidewire with a stiff type guidewire. 7. Insert a $5 \mathrm{Fr}$ or $6 \mathrm{Fr}$ tip pigtail-type transnasal biliary drainage tube or $7 \mathrm{Fr}$ pigtail-type bile duct plastic stent in the gallbladder. 8. Endoscopic sphincterotomy (EST) may be added because of concerns over possible obstructive pancreatitis when inserting a stent with a diameter larger than 7 Fr. Keeping in mind that possible pancreatitis may occur with a transpapillary approach, the procedure should be completed promptly without sticking in difficult cases, although this is often difficult; however, a transhepatic approach may also be considered.

Under such circumstances, the EUS-GBD technique has attracted attention in recent years [10]; however, it has not yet been standardized. In future, with device development and establishment of endoscopic procedures, EUS-GBD may become the preferred treatment for AC for endoscopists accustomed to EUS intervention.

\section{Competing interests}

None

References

[1] Yokoe M, Hata J, Takada T et al. Tokyo Guidelines 2018: diagnostic criteria and severity grading of acute cholecystitis (with videos). J Hepatobiliary Pancreat Sci 2018; 25: 41 - 54

[2] Okamoto K, Suzuki K, Takada T et al. Tokyo Guidelines 2018: flowchart for the management of acute cholecystitis. J Hepatobiliary Pancreat Sci 2018; 25: 55-72

[3] Mori Y, Itoi T, Baron TH et al. Tokyo Guidelines 2018: management strategies for gallbladder drainage in patients with acute cholecystitis (with videos). J Hepatobiliary Pancreat Sci 2018; 25: 87-95

[4] Wakabayashi G, Iwashita Y, Hibi T et al. Tokyo Guidelines 2018: surgical management of acute cholecystitis: safe steps in laparoscopic cholecystectomy for acute cholecystitis (with videos). J Hepatobiliary Pancreat Sci 2018; 25: 73-86

[5] Itoi T, Takada T, Hwang TL et al. Percutaneous and endoscopic gallbladder drainage for acute cholecystitis: international multicenter comparative study using propensity score-matched analysis. J Hepatobiliary Pancreat Sci 2017; 24: 362-368

[6] Itoi T, Coelho-Prabhu N, Baron TH. Endoscopic gallbladder drainage for management of acute cholecystitis. Gastrointest Endosc 2010; 71 : $1038-1045$

[7] Itoi T, Sofuni A, Itokawa F et al. Endoscopic transpapillary gallbladder drainage in patients with acute cholecystitis in whom percutaneous transhepatic approach is contraindicated or anatomically impossible (with video). Gastrointest Endosc 2008; 68: 455-460

[8] Nakahara K, Michikawa Y, Morita R et al. Endoscopic transpapillary gallbladder stenting using a newly designed plastic stent for acute cholecystitis. Endosc Int Open 2019; 07: E1105-E1114

[9] Itoi T, Kawakami H, Katanuma A et al. Endoscopic nasogallbladder tube or stent placement in acute cholecystitis: a preliminary prospective randomized trial in Japan (with videos). Gastrointest Endosc 2015; 81: $111-118$

[10] Itoi T. New era in acute cholecystitis treatment: encouraging results for interventional endoscopists. Gastrointest Endosc 2017; 85: 88 89 